\title{
Combining instance and feature neighbours for extreme multi-label classification
}

\author{
Len Feremans ${ }^{1}$ - Boris Cule ${ }^{2}$ - Celine Vens ${ }^{3,4}$ - Bart Goethals ${ }^{1,5}$
}

Accepted: 26 February 2020

\begin{abstract}
Extreme multi-label classification problems occur in different applications such as prediction of tags or advertisements. We propose a new algorithm that predicts labels using a linear ensemble of labels from instance- and feature-based nearest neighbours. In the feature-based nearest neighbours method, we precompute a matrix containing the similarities between each feature and label. For the instance-based nearest neighbourhood, we create an algorithm that uses an inverted index to compute cosine similarity on sparse datasets efficiently. We extend this baseline with a new top- $k$ query algorithm that combines term-at-a-time and document-at-a-time traversal with tighter pruning based on a partition of the dataset. On ten real-world datasets, we find that our method outperforms state-of-the-art methods such as multi-label $k$-nearest neighbours, instance-based logistic regression, binary relevance with support vector machines and FastXML on different evaluation metrics. We also find that our algorithm is orders of magnitude faster than these baseline algorithms on sparse datasets, and requires less than $20 \mathrm{~ms}$ per in-
\end{abstract}

\footnotetext{
凶len.feremans@uantwerpen.be

boris.cule@uantwerpen.be

\ celine.vens@kuleuven.be

\bart.goethals@uantwerpen.be

${ }^{1}$ Department of Computer Science, Universiteit Antwerpen, Belgium

2 Department of Accountancy and Finance, Universiteit Antwerpen, Belgium

3 Faculty of Medicine, Katholieke Universiteit Leuven, Belgium

4 ITEC, imec research group at Katholieke Universiteit Leuven, Belgium

5 Monash University, Melbourne, Australia
}

A preliminary version of this paper was published as "Combining instance and feature neighbors for efficient multi-label classification" at DSAA 2017 [1]. stance to predict labels for extreme datasets without the need for expensive hardware.

Keywords Extreme multi-label classification · Itembased collaborative filtering $\cdot k$-nearest neighbours . Top- $k$ queries $\cdot$ Information retrieval

\section{Introduction}

Multi-label classification problems occur in a large variety of domains, such as text categorization, where a document has multiple categories, scene classification, where various regions of an image have a label, and bioinformatics, where we are interested in predicting numerous functions for a gene. In this work, we consider sparse datasets that occur naturally in these domains, i.e., where features correspond to patterns, such as term frequency-inverse document scores for word occurrences in texts or clusters in images.

Two main strategies exist for solving the multi-label task. The first strategy is to reduce the multi-label problem into a combination of single-label problems. The binary relevance method ignores label dependencies and trains a separate model to predict each label independently of other labels using one-versus-all sampling [2]. Classifier chains approximate label dependencies, but also require to train a separate model for each label [3]. If the set of labels $L$ is large, training $|L|$ different models using binary relevance or classifier chains is not scalable. A second strategy is to adapt existing single-label classifiers to output multiple labels. Wellknown adaptations of single-label classification techniques have been made to adaBoost, decision trees, support vector machines, $k$-nearest neighbour and others $[4-7]$. 
Trending challenges in multi-label classification research include methods that account for possible dependencies between labels, deal with label skew (where most labels are only covered by a few instances), and consider the computational cost of building a model [8]. Extreme multi-label classification is an active research topic that considers the computational cost of generating a model when the number of labels is very high. Recently several methods have been proposed that try to address these challenges. These methods reduce the dimensionality of the label space or build a hierarchical ensemble of tree-based models, such as FASTXML, where the number of models to train is logarithmic in the number of labels [9-11]. While these approaches are accurate and fast at testing time, they require significant resources at training time. Moreover, each of these methods needs to tune many hyperparameters for optimal performance.

Related to multi-label classification is the field of recommender systems. Here, the task is to rank items a user might click, often based on past preferences. Two well-known recommender systems are user-based and item-based collaborative filtering [12-14]. An advantage of both approaches is that the results can be explained, i.e., using "people who liked this item also liked" type of explanations. Applying these techniques for multi-label classification is a major goal of this work.

User-based collaborative filtering is a memory-based learning algorithm where we need to compute the nearest neighbours. The problem of finding the exact set of $k$-nearest neighbours is studied under different names: all pairs similarity search, top- $k$ set similarity joins, $k$ nearest neighbour graph construction and top- $k$ queries [15-21]. We propose a new algorithm to compute the exact $k$-nearest neighbours using pruning. Similar to search problems in information retrieval, we want to find a set of instances in our training dataset that is the most similar to a test instance for which we wish to predict labels. A key difference with information retrieval is that our test instances, or queries, typically have many more non-zero feature values than is usual in search, which has a severe impact on performance. Therefore, we adapt research from information retrieval and create a new top- $k$ query algorithm. Technically, we combine term-at-a-time and document-at-a-time traversal using Weak-AND pruning [22]. Different from the previous work in information retrieval, our primary reason for first traversing the instances using term-at-a-time, is based on finding proper constraints such that more candidate instances get pruned using a tighter upperbound [21].

In this work, we make the following contributions. First, we implement instance-based $k$-nearest neighbours, an adaptation of user-based collaborative filtering, for multi-label classification. Next, we implement the feature-based $k$-nearest neighbours method, an adaptation of item-based collaborative filtering, that computes the nearest labels for each feature in a column-wise manner. Finally, we combine both instance- and feature-based neighbourhood predictions using a linear ensemble. Second, we make the $k$-nearest neighbours search scalable for sparse datasets with an extremely high number of labels, features and instances. The baseline method uses an inverted index and organises computation so that we only perform non-zero similarity term computations, which we improve with a top- $k$ query algorithm.

We validate the accuracy of our method on 10 realworld datasets and compare with multi-label classification methods such as multi-label $k$-nearest neighbours, instance-based logistic regression, binary relevance with support vector machines as a base learner and FASTXML on different evaluation metrics $[7,11,23]$. We also compare the pruning ability and runtime performance of our $k$-nearest neighbours algorithm with state-of-theart top- $k$ query retrieval methods, such as term-at-a-time traversal, in-memory document-at-a-time traversal with Weak-AND pruning and Fagin's threshold algorithm [19, 21, 22]. Compared to the original version of this paper [1], we improve our algorithms for making predictions in different ways and propose a new algorithm to compute the nearest neighbours more efficiently based on top- $k$ queries.

The remainder of this paper is organised as follows. In Section 2 we define the problem setting. In Section 3 we describe our algorithm for multi-label classification. In Section 4 we describe our method for finding the $k$ nearest neighbours more efficiently. We experimentally validate our method and compare it with existing stateof-the-art methods in Section 5 and discuss related and future work in Section 6. Finally, we conclude in Section 7.

\section{Problem Setting}

Definition 1 (Multi-label Dataset) Let $X \in \mathbb{R}^{N \times M}$ denote the set of training points and let $Y \in\{0,1\}^{N \times L}$ denote the set of labels. The training dataset $\mathcal{D}$ consists of $N$ instances $\mathcal{D}=\left\{\left(x_{1}, \mathbf{y}_{\mathbf{1}}\right), \ldots,\left(x_{N}, \mathbf{y}_{\mathbf{N}}\right)\right\}$ where each $M$-dimensional feature vector $x_{i} \in \mathbb{R}^{M}$ is associated with an L-dimensional label vector $\mathbf{y}_{\mathbf{i}} \in\{0,1\}^{L}$.

We assume that feature values are transformed into real numbers higher than or equal to zero.

Definition 2 (Cardinality) We define feature cardinality as the average number of non-zero features for 
each instance. Analogously, we define label cardinality as the average number of labels for each instance, that is:

$$
\begin{gathered}
f \operatorname{card}(\mathcal{D})=\frac{1}{N} \sum_{i=1}^{N} \sum_{j=1}^{M} \delta\left(x_{i, j}\right), \\
\operatorname{lcard}(\mathcal{D})=\frac{1}{N} \sum_{i=1}^{N} \sum_{j=1}^{L} y_{i, j},
\end{gathered}
$$

where $\delta\left(x_{i, j}\right)$ is 1 if $x_{i, j}>0$ and 0 otherwise and $y_{i, j}$ is the binary value of label $j$ for instance $i$.

Definition 3 (Column) We use $f_{j}=\left\{x_{1, j}, \ldots, x_{N, j}\right\}$ $\in \mathbb{R}^{N}$ to denote the column of values for feature $j$. Likewise we use $l_{j}=\left\{y_{1, j}, \ldots, y_{N, j}\right\} \in\{0,1\}^{N}$ to denote the column of values for label $j$.

Definition 4 (Density) We define feature and label density as

$$
\begin{aligned}
\operatorname{fdens}\left(f_{j}\right) & =\frac{\left|\left\{x_{i, j} \mid x_{i} \in X \wedge x_{i, j} \neq 0\right\}\right|}{N}, \\
\operatorname{ldens}\left(l_{j}\right) & =\frac{\left|\left\{y_{i, j} \mid y_{i} \in Y \wedge y_{i, j} \neq 0\right\}\right|}{N} .
\end{aligned}
$$

For sparse datasets, we observe that feature and label cardinality are small compared to $M$ and $L$, and that there is a skewed distribution where only a few features (or labels) have a high density, and most features (or labels) have a density close to 0 .

Problem 1 The task for multi-label classification is to predict a subset of labels for each test instance $x_{q} \in$ $X_{\text {test }}$ for which the set of labels $\mathbf{y}_{\mathbf{q}}$ is unknown. Formally we have to learn a function $h: X \rightarrow\{0,1\}^{L}$ that optimises a selected evaluation metric.

The function $h$ can be implemented as $h(x)=t(f(x))$ where $f$ produces a confidence (or probability) score for each label and $t$ is a threshold function.

\section{Linear Combination of Instance- and Feature-based kNN}

Our classification method consists of instance-based $k$ nearest neighbours $(\mathrm{kNN})$, feature-based $\mathrm{kNN}$ and the linear combination of both predictions.

\subsection{Instance-based kNN}

The algorithm begins by searching for the $k$-nearest neighbours $x_{i}$ in the training data for each test (or query) instance $x_{q}$ using cosine similarity.
Definition 5 (Instance-based Cosine Similarity) The cosine similarity is defined as

$$
\operatorname{sim}_{\mathrm{INS}}\left(x_{q}, x_{i}\right)=\frac{x_{q} \cdot x_{i}}{\left\|x_{q}\right\|_{2} \cdot\left\|x_{i}\right\|_{2}}=x_{q} \cdot x_{i},
$$

where we make sure that all instances are normalised to unit length during preprocessing.

Definition 6 (Instance-based Confidence Score) To compute the confidence score for instance $x_{q}$ for ( $a$ single) label $y_{j}$ we define the following function:

$$
\hat{y}_{q, j}^{\mathrm{INS}}=\frac{\sum_{x_{i} \in \mathrm{KNN}\left(x_{q}\right)} y_{i, j} \cdot \operatorname{sim}_{\mathrm{INS}}\left(x_{q}, x_{i}\right)^{\alpha}}{\sum_{x_{i} \in \mathrm{KNN}\left(x_{q}\right)} \operatorname{sim}_{\mathrm{INS}}\left(x_{q}, x_{i}\right)^{\alpha}} .
$$

This function is an adaption of user-based collaborative filtering, where the similarity in feature values replaces the similarity between user preferences, and we do not recommend an item but a label [24]. Also, we apply a power transformation to the similarities. For example, if we apply the power $\alpha=2$, we give similarities closer to 1 more weight compared to similarities closer to 0.01 . Vice versa, $\alpha=0.5$ has the reverse effect.

Algorithm We retrieve the $k$-nearest neighbours by using an inverted index (IID) and compute only nonzero terms for similarity. CREATEINDEX is shown in Algorithm 1. We associate each feature with a set of (training) instances and their non-zero feature value.

In InSTANCEKNNSEARCH, shown in Algorithm 2, we compute the cosine similarity between $x_{q}$ and all instances incrementally thereby only computing non-zero terms of each dot product. We first loop over each nonzero feature $x_{q, j}$, then fetch all candidates $x_{i}$ that have a (non-zero) $x_{i, j}$ value from the IID and then increment the partial dot product $x_{q, j} \cdot x_{i, j}$. Finally, we use partial sort, i.e., using heap sort, to maintain the top $k$ instances with the highest cosine similarity.

We compute the prediction scores for each label using InstancEKnnPREDICT, shown in Algorithm 3. We only compute predictions for labels that are present in

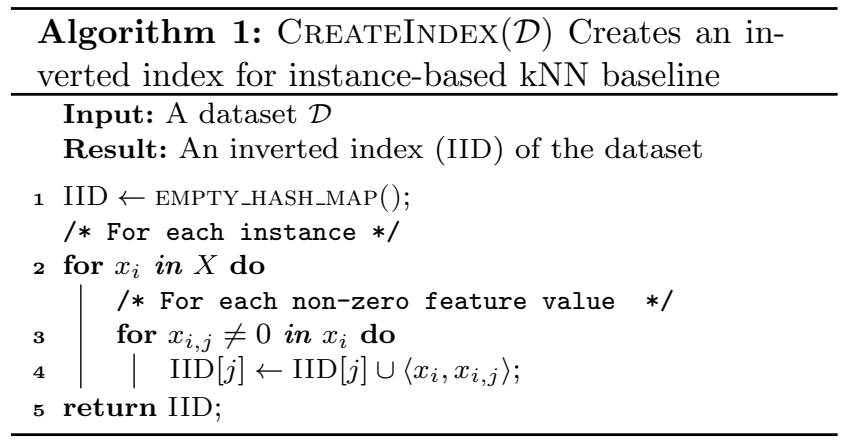



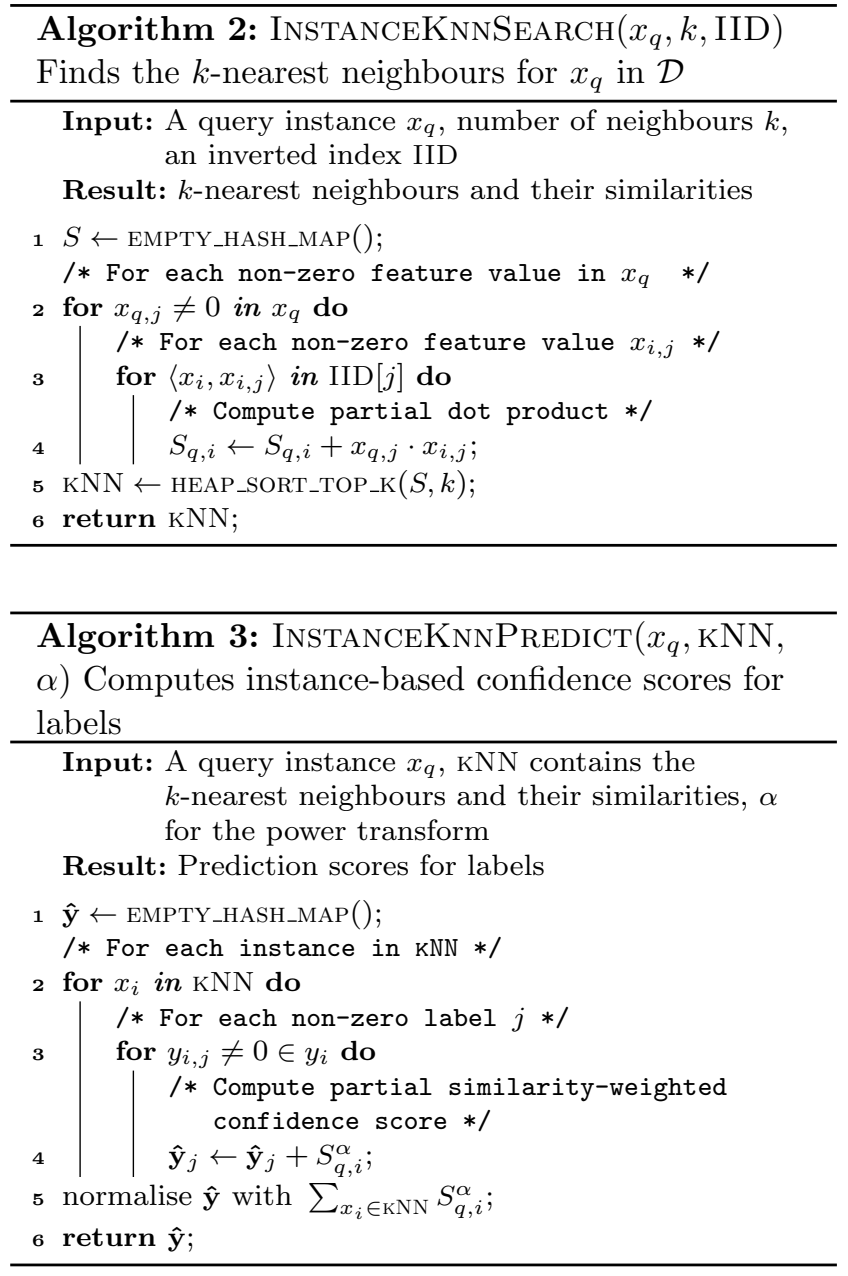

any of the $k$-nearest neighbours. As in INSTANCEKNNSEARCH, we organise the computation so that we only compute non-zero increments to each label score. Remark that in our implementation, we compute similarities and predictions in parallel. We initialise shared hash tables statically, so subsequent updates to partial scores (or similarities) from different threads can occur in a lock-free manner [25]. This results in performance gains almost linear with the number of processors.

Complexity For instance-based kNN search the complexity is $\mathcal{O}(N \times M)$, but in practice, we observe that the average runtime is closer to $\mathcal{O}(\tilde{n} \times \tilde{m})$ for sparse datasets. Here $\tilde{n}$ is proportional to the average number of candidate instances, i.e., instances fetched from the inverted index, and $\tilde{m}$ is proportional to the feature cardinality. We will analyse the runtime of this algorithm in Section 5. We remark that the expensive neighbourhood search is performed once and is independent of the number of labels $L[26]$.

Hyperparameter Optimalisation An essential advantage of our method is that for optimising $k$ using grid search we need to compute the nearest neighbours only once. First, we search for the nearest neighbours with a maximal value of $k_{\max }$. For smaller values of $k$, we just take the first $k$ values of the cached $k_{\text {max }}$-nearest neighbours. We argue that this makes $k$ a virtual hyperparameter, meaning that we optimise it efficiently on a validation set.

Second-order Instance Variation A possible disadvantage of the instance-based $\mathrm{kNN}$ method is that we ignore inter-label dependencies: the prediction for a given label is obtained independently of the values of other labels. We propose an extension that uses the second-order neighbourhood to handle this situation. For details, we refer to our original paper [1].

\subsection{Feature-based kNN}

Feature-based kNN is an adaptation of item-based collaborative filtering for multi-label classification [14].

\section{Definition 7 (Feature-based Cosine Similarity)}

For feature-based predictions, we compute the cosine similarity between each feature column $f_{i}$ and each label column $l_{j}$ using,

$$
\operatorname{sim}_{\mathrm{FL}}\left(f_{i}, l_{j}\right)=\frac{f_{i} \cdot l_{j}}{\left\|f_{i}\right\|_{2} \cdot\left\|l_{j}\right\|_{2}}=f_{i} \cdot l_{j},
$$

where we make sure that all feature and label vectors are normalised to unit length during preprocessing.

\section{Definition 8 (Feature-based Confidence Score)}

We compute the confidence score for a test instance $x_{q}$ and a label $y_{j}$ using:

$$
\hat{y}_{q, j}^{\mathrm{FL}}=\frac{\sum_{i=1}^{M} x_{q, i} \cdot \operatorname{sim}_{\mathrm{FL}}\left(f_{i}, l_{j}\right)^{\beta}}{\sum_{i=1}^{M} x_{q, i}},
$$

where we apply the power $\beta$ to the similarities.

We compute the full similarity matrix between all pairs of feature and label columns at training time. When $L$ is extremely large, we consider a variation to featurebased $\mathrm{kNN}$, that only computes a (non-zero) prediction for labels that occur at least once in the neighbourhood, i.e.,

$$
y_{j} \in \bigcup_{x_{q, i} \in x_{q} \wedge x_{q, i} \neq 0} \operatorname{KNN}\left(f_{i}\right),
$$

which is more scalable given an extreme number of labels.

Algorithm For feature-based kNN we first compute a matrix containing the similarities between all features and labels in $\mathcal{D}$. We use sparse data structures 

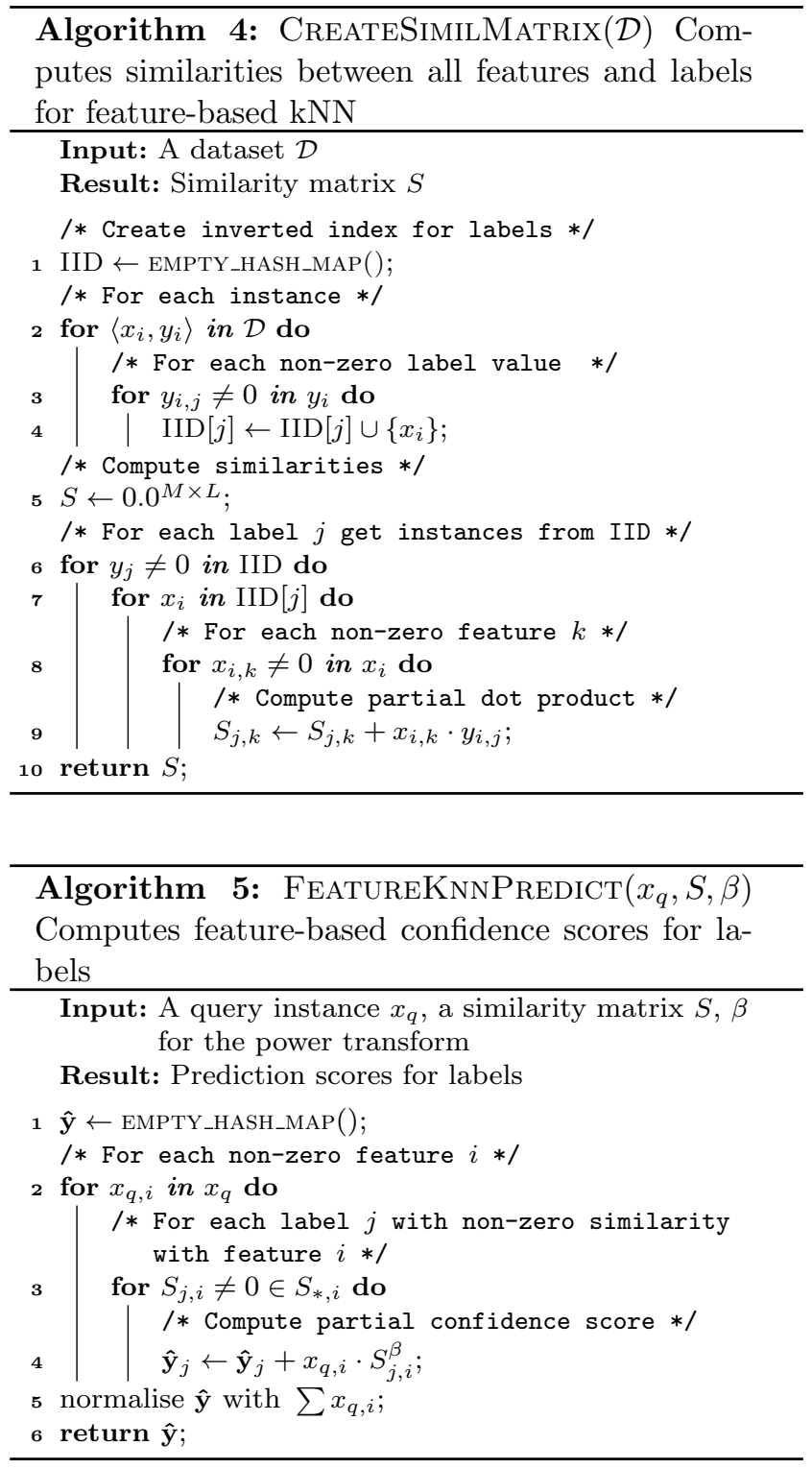

as we assume most values for label column $y_{j}$ and feature column $f_{k}$ will be 0 . We use a technique similar to INSTANCEKNNSEARCH and compute the feature-based cosine similarity incrementally. The function CREATESimilMatrix is shown in Algorithm 4. First, we create an index that associates each of the $L$ labels with a set of positive instances. After indexing, we fetch positive instances $x_{i}$ for each label. For each instance $x_{i}$, we traverse over each non-zero feature $x_{i, k}$ and compute a non-zero term of the dot product between label $y_{j}$ and feature $f_{k}$.

FeatureKnnPredict is shown in Algorithm 5. We follow a similar approach as INSTANCEKNNPREDICT to only compute non-zero terms of each confidence score. We remark that we also experimented with an alternative confidence score that considers the $k$ nearest fea- tures for each label, but in preliminary experiments, this did not increase average results while requiring an extra hyperparameter.

Complexity Computing the similarity matrix has a complexity of $\mathcal{O}\left(\frac{1}{2} M \times L \times N\right)$. However, in practice runtime is closer to $\mathcal{O}\left(\frac{1}{2} M \times \tilde{l} \times \tilde{n}\right)$ for sparse datasets. Here $\tilde{l}$ is proportional to the average number of candidate labels, that is the number of labels sharing at least one instance with each feature and $\tilde{n}$ is proportional to the average number of non-zero feature values (or labels) column-wise. We observe that the similarity matrix can be computed once at training time for all test instances, while at test time only prediction scores have to be computed. This makes feature-based $\mathrm{kNN}$ very efficient and is arguably one of the reasons why item-based collaborative filtering is so popular in realworld web applications.

\subsection{Linear Combination}

We introduce a straightforward ensemble method based on the Linear Combination of the confidence scores of the Instance- and Feature-based $k$-nearest neighbours (LCIF). Combinations of the two techniques have been studied in collaborative filtering research, but not in multi-label classification [24, 27].

Definition 9 (LCIF Confidence Score) We compute the confidence score for test instance $x_{q}$ for label $y_{i}$ using

$$
\hat{y}_{q, i}=\lambda \hat{y}_{q, i}^{\mathrm{INS}}+(1-\lambda) \hat{y}_{q, i}^{\mathrm{FL}},
$$

where $\lambda \in[0,1]$ is a hyperparameter that is optimised on a validation sample for each evaluation metric.

For datasets with many labels we compute this score only for candidate labels, that is, labels $i$ that have a non-zero score for either $\hat{y}_{q, i}^{\mathrm{INS}}$ or $\hat{y}_{q, i}^{\mathrm{FL}}$. The main LCIF algorithm is shown in Algorithm 6.

\subsection{Thresholding}

To obtain a set of predicted labels, we apply a single threshold $[3,28]$.

Definition 10 (Single Threshold) Given confidence scores $\hat{y}_{q, j}$ for instance $x_{q}$ and each label $y_{j}$, we predict a set of labels using a single threshold $t$ :

$$
h\left(x_{q}\right)=\left\{y_{j} \mid \hat{y}_{q, j}>=t\right\}, \forall y_{j} \in L .
$$




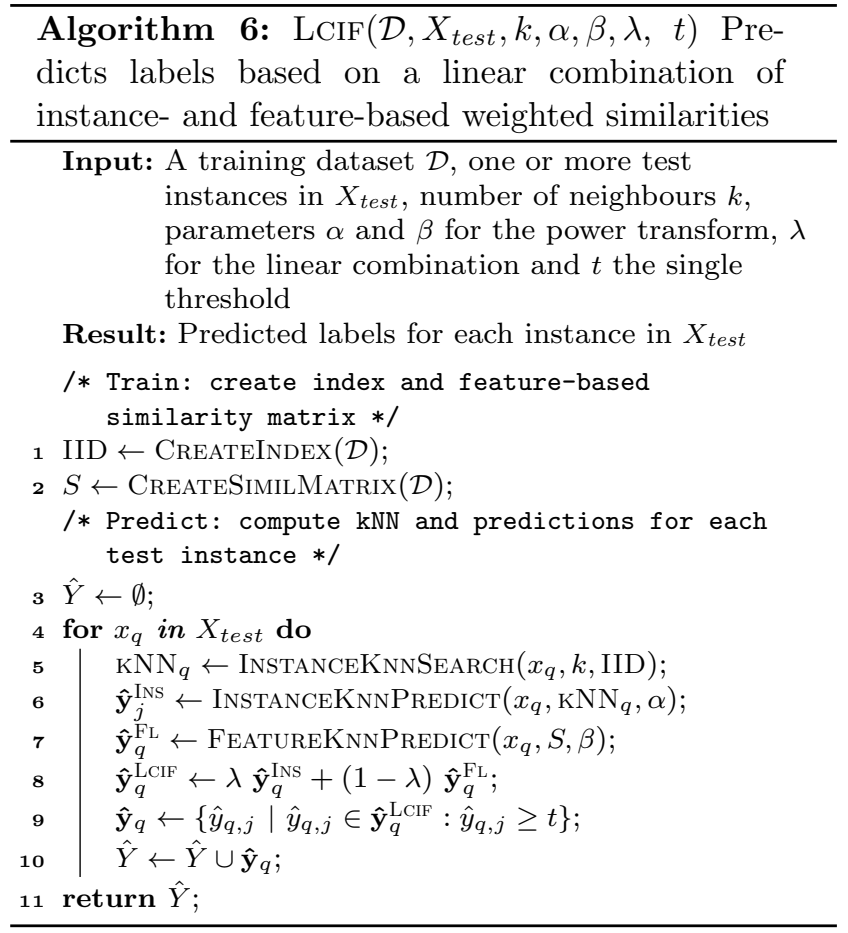

We determine $t$ automatically by selecting the value of $t$ that minimises the difference in label cardinality between the actual and predicted label set. That is,

$$
\underset{t}{\operatorname{argmin}}\left|\frac{1}{N} \sum_{i=1}^{N} \sum_{j=1}^{L} \delta\left(\hat{y}_{i, j}>t\right)-\operatorname{lcard}(\mathcal{D})\right|,
$$

where $\delta\left(\hat{y}_{i, j}>t\right)$ returns 1 if the confidence score is higher than $t$ and 0 otherwise. Alternatively, we make use of a label-specific threshold [29,30]. This allows us to lower the threshold for minority labels in imbalanced datasets.

Definition 11 (Label-specific threshold) We predict a set of labels using a separate threshold $t_{y_{j}}$ for each label $y_{j}$ :

$$
h\left(x_{q}\right)=\left\{y_{j} \mid \hat{y}_{q, j}>=t_{y_{j}}\right\}, \forall y_{j} \in L
$$

We remark that for many multi-label datasets, there is at least one label for every instance. Therefore, if the highest-scoring label is below the threshold, we ignore the threshold value [26].

\section{Fast kNN Search}

A problem with the instance-based $\mathrm{kNN}$ search is that because of its inherent $O(N)$ complexity to search for the $k$-nearest neighbours, it does not scale to extreme datasets. In the previous section, we created a baseline algorithm optimised for sparse datasets. In this section, we improve on this baseline. This is important in interactive applications since we must perform the search at test time and every millisecond is important.

\subsection{Indexing}

The problem of instance-based $\mathrm{kNN}$ is similar to top- $k$ queries algorithms in information retrieval. A key difference, however, is that search queries are typically much shorter, having less than 10 terms. Real-world search engines often limit search queries to 50 terms. In our case, each query is a test instance, that consists of many more non-zero dimensions on average. We will show that existing state-of-the-art top- $k$ query algorithms are less efficient in this setting. Therefore, we propose a new top- $k$ query algorithm for computing the exact set of $k$-nearest neighbours ranked on cosine similarity.

Top-k Query Algorithm Our method is an extension of the work of Fontoura et al. that combines two ways to traverse the most relevant instances (or documents) given a certain test instance (or query): Document-ata-time (DAAT) and Term-at-a-time (TAAT) [21]. Using this framework, Algorithm 2 computes cosine similarity following a TAAT strategy, that is, for every nonzero feature, or term, of a test instance we fetch all instances, or documents, from the inverted index and increment the partial similarity of each document with the non-zero weight in that dimension. In DAAT traversal we keep all documents in the inverted index sorted on document order and traverse through all posting lists (the inverted index for each term) simultaneously similar to a merge join. Using this document-per-document manner, we can compute the complete similarity score for each document in turn. We propose to first traverse using TAAT and next using DAAT. We focus on memory-resident indexes, thereby assuming that memory in present-day is often large enough to maintain the complete index [21].

Partitioning We observe that in real-world datasets feature values in most dimensions have a high standard deviation. For example, we can encode text documents using a bag-of-words encoding with term frequencyinverse document frequency and get a significant difference in values between terms that frequently occur in one document but seldom occur in others, and words that are infrequent in one document and frequent overall. This variation is a useful property that we exploit. 


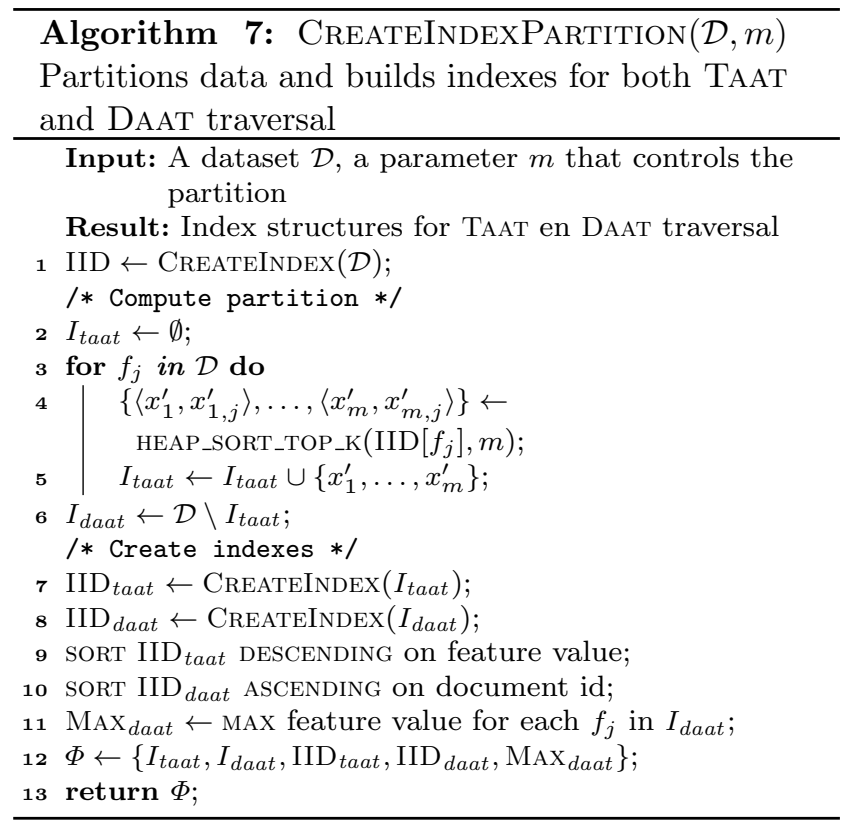

Definition 12 (Partition) First, we partition all instances into two disjoint sets:

$$
\begin{gathered}
I_{\text {taat }}=\left\{x_{i} \mid x_{i} \in \mathcal{D} \wedge \exists x_{i, j} \in x_{i}: \operatorname{rank}\left(x_{i, j}, f_{j}\right) \leq m\right\}, \\
I_{\text {daat }}=\mathcal{D} \backslash I_{\text {taat }},
\end{gathered}
$$

where we use $\operatorname{rank}\left(x_{i, j}, f_{j}\right) \leq m$ to denote that feature value $x_{i, j}$ is ranked before place $m$ in the (descending) ordered posting list of feature $j$.

The partition strategy has three useful consequences. Firstly, by using the partitioned and sorted inverted index, we encounter high feature values first during TAAT traversal and are more likely to find instances with a high cosine similarity early on. This is important since we prune instances during DAAT if the similarity cannot be higher than the $k^{\text {th }}$ candidate after TAAT traversal. Secondly, we use Weak-AND (WAND) for pruning during DAAT traversal [22]. The WAND upper bound depends on the maximum feature value in each dimension. Because of the partitioning, we guarantee that this maximum is smaller than the first $m$ values. Thirdly, by having two disjoint partitions, additional overhead for pruning and index creation is minimal.

\footnotetext{
Algorithm The algorithm CREATEIndexPartition for both TAAT and DAAT index creation is shown in Algorithm 7 and consists of two phases. In the first phase, we create an inverted index for all documents and compute the partition of all instances. For each feature or term, we find the instances with the $m$ highest feature values and add these instances to $I_{\text {taat }}$. We can compute this efficiently using heap sort on the posting list for each term. In the second phase, we make a
}

complete TAAT index by adding all feature values for all instances in $I_{\text {taat }}$. Remark that this index also includes feature values that are not in the top- $m$. The rationale for making the index complete is that we can compute the full similarity for each instance in $I_{\text {taat }}$ without resorting to less efficient random access operations. Finally, we add all remaining documents to the DAAT index sorted on document ID. We also maintain the maximal feature value in each posting list. In our implementation, we keep the feature values local to the index as we want to avoid cache misses. We remark that in practice we set the parameter $m$ to a small value, e.g., between 1 and 25 for large datasets and closer to 100 for extreme datasets. Alternatively, $m$ could be defined relatively as the percentage of all documents in $I_{\text {taat }}$.

4.2 Fast $k$-nearest Neighbour Search using Term- and Document-at-a-time with Weak-And Pruning

Pruning In essence DAAT is a merge join over the different posting lists sorted on document ID. We can, however, skip instances based on an upper bound using the Weak-And iterator [22].

Definition 13 (Upper Bound Cosine Similarity) Given a query $x_{q}$ we compute an upper bound on each cosine similarity term:

$$
U B_{q, j}=\max \left(\left\{x_{i, j} \mid x_{i} \in I_{d a a t}\right\}\right) \cdot x_{q, j} .
$$

For any $x_{i} \in I_{\text {daat }}$ it holds that

$$
\operatorname{sim}_{\mathrm{INS}}\left(x_{q}, x_{i}\right) \leq \sum_{x_{q, j} \in x_{q}} U B_{q, j} .
$$

Therefore, we prune instances without computing the full similarity if

$$
\sum_{x_{q, j} \in x_{q} \wedge x_{i, j} \neq 0} U B_{q, j} \leq \sum_{x_{q, j} \in x_{q}} U B_{q . j} \leq \theta,
$$

where $\theta$ is the $k^{\text {th }}$ largest similarity of instances already visited during TAAT and the ongoing DAAT traversal. Remark that for most instances $x_{i}$ only a small subset of the features of $x_{q}$ will also be non-zero in $x_{i}$.

Algorithm The main algorithm for finding the exact $k$-nearest neighbours is shown in Algorithm 8. INSTANCEKNNFAST consists of two phases. We start by computing the $k$ nearest neighbours of all instances in $I_{\text {taat }}$ using TAAT by calling INSTANCEKNNSEARCH. We then add these instances to a heap. A heap is more efficient for managing the current instances as we want to access the current $k^{\text {th }}$ maximal similarity efficiently. In 
the second phase, we traverse candidate instances using DAAT (line 3-20) and our extension to WAND pruning. We start by initialising the upper bound for $x_{q}$ by computing the dot product between every non-zero feature value $x_{q, j}$ and the precomputed value $\operatorname{MAx}_{\text {daat }}[j]$. Next, we iterate over instances in $I_{\text {daat }}$ that have at least one feature shared with $x_{q}$ (as determined by $\left.\mathrm{IID}_{\text {daat }}\right)$. We start our while loop with the first document $\left(\right.$ offsets $\left._{j}=0\right)$ in each posting list and order these instances on ascending ID (line 8-11).

If $x_{k_{1}}$ is the document with the smallest ID for any posting list then for any other document $x_{k_{2}}$, with $k_{2}>k_{1}$, we know that $x_{k_{1}}$ has a zero value in that posting list. Therefore, we prune $x_{k_{1}}$ if $U B_{q, k_{1}}$ is smaller than $\theta$. Likewise, we prune $x_{k_{2}}$ if $U B_{q, k_{1}}+U B_{q, k_{2}}$ is smaller than $\theta$, etc. We increment the upper bound $U B_{\text {cur }}$ in a feature-by-feature manner and prune any documents that are below this accumulated value (line 12-17). We stop when a pivot document is identified, meaning the first document that is higher than the upper bound. We then compute the full similarity for the pivot instance and add it to the heap, where it will replace a candidate if the similarity is higher (line 19). Finally, we advance each posting list to the next document. If the next document identifier is larger than the pivot, we do not update the offset. Otherwise, we ad-

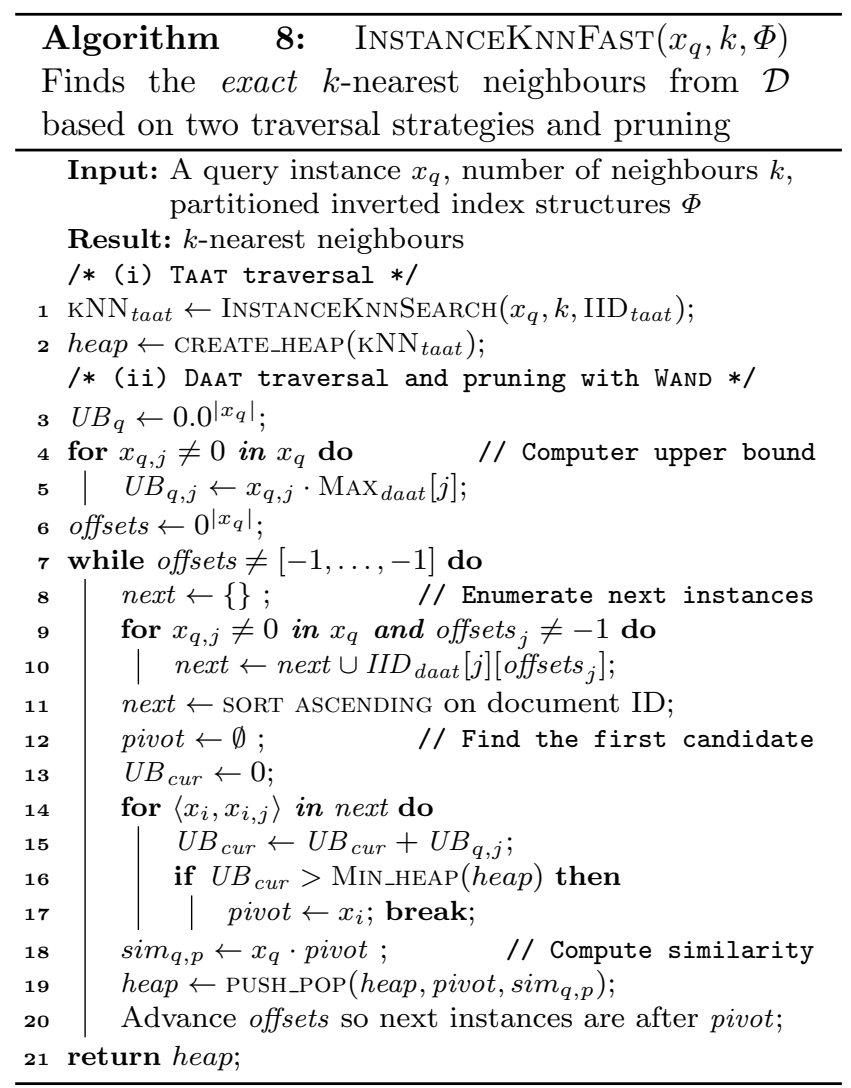

vance the posting list to point to a document with an identifier after the current pivot. In the worst case, the pivot document is always the first candidate, and we advance by one document at a time. In the best case, however, there are $\left|x_{q}\right|$ documents and the pivot document is the last document. Then we can advance by $\left|x_{q}\right|-1$ documents, thereby pruning these documents without computing the full similarity.

\section{Experiments}

We now study the accuracy and efficiency of LCIF and INSTANCEKNNFAST.

\subsection{Experimental Setup}

Datasets We have selected five large and five extreme datasets. Table 1 shows the most important characteristics of each dataset. The datasets are available in well known multi-label repositories [31-33]. The Medical dataset consists of nearly 1000 documents containing free clinical text, originally collected at a children's hospital medical centre's department of radiology. The problem is to assign one or more medical diagnoses or procedures coded using ICD-9-CM based on free clinical text. The documents are represented using a sparse bagof-words encoding. The Corel5k dataset is a scene classification dataset. Labels represent familiar concepts such as sea, sky, cat or forest. The images are represented using 499 binary features. A feature value of 1 indicates that a certain segment in the image belongs to a certain cluster. The Bibtex dataset represents a tag assignment problem. The Delicious dataset is similar to Bibtex. Wiki10 corresponds to 20000 Wikipedia articles. For these three datasets, the labels (or tags) were assigned using the social tagging sites Bibsonomy and Del.icio.us. Note that the label cardinality with social tagging is higher. In the $I M D B-F$ dataset, the task is to assign one or more of the 28 movie genres, based on movie summary texts from IMDB. This dataset is larger, containing more than 100000 summaries. However, there are only 28 movie genres, and the total dictionary of terms is limited.

The extreme Eurlex dataset is a collection of documents about European law and has close to 4000 categories. Reuters Corpus Volume I ( $R C V 1)$ is a benchmark dataset for text categorisation containing more than 700000 labelled news articles made available by the press agency Reuters. AmazonCat contains over a million instances of Amazon products with labels and reviews. We selected the version that has more than 13000 labels. Finally, WikiLSHTC consists of more than 
Table 1 Characteristics of five large and five extreme multi-label datasets

\begin{tabular}{lrrrrrrrr} 
Dataset & Train & Test & Features & Labels & lcard & fcard & $\begin{array}{c}\text { Avg. } \\
\text { ldens }\end{array}$ & $\begin{array}{c}\text { Avg. } \\
\text { fdens }\end{array}$ \\
\hline Medical & $N$ & $N_{\text {test }}$ & $M$ & $L$ & & & \\
Corel5k & 333 & 645 & 1449 & 45 & 1.2 & 13.4 & 0.0277 & 0.0092 \\
Bibtex & 5000 & 500 & 499 & 374 & 3.5 & 8.3 & 0.0094 & 0.0166 \\
Delicious & 4880 & 2515 & 1836 & 159 & 2.4 & 68.7 & 0.0151 & 0.0374 \\
IMDB-F & 12920 & 3185 & 500 & 983 & 19.1 & 18.3 & 0.0193 & 0.0366 \\
Eurlex & 72551 & 48368 & 1001 & 28 & 2.0 & 19.4 & 0.0714 & 0.0194 \\
Wiki10 & 15539 & 3809 & 5000 & 3956 & 5.3 & 237.0 & 0.0013 & 0.0474 \\
RCV1 & 14147 & 6617 & 101890 & 30940 & 18.6 & 669.0 & 0.0006 & 0.0065 \\
AmazonCat & 118623947 & 155962 & 46672 & 2456 & 4.8 & 74.0 & 0.0019 & 0.0016 \\
WikiLSHTC & 1778352 & 586782 & 203873 & 13330 & 5.1 & 71.1 & 0.0004 & 0.0003 \\
\hline
\end{tabular}

a million instances and features and 325000 categories. The source is Wikipedia. A large number of features is due to the large corpus size. For this dataset, there is also a hierarchy between the labels available which we ignore. The dataset originates from the large-scale hierarchical text classification challenge [34]. Eurlex, Wiki10, RCV1, AmazonCat and WikiLSHTC are extreme datasets since they have thousands of labels [33]. We remark that although the extreme datasets contain more labels and features, they are also extremely sparse and the cardinality of labels and features remains comparable to the large datasets. A key advantage of our method is that it is optimised for sparse datasets.

State-of-the-art Methods For the large datasets, we compare the performance of the instance- and featurebased $\mathrm{kNN}$ methods and their linear combination LCIF with the following state-of-the-art algorithms. Multilabel $k$-nearest neighbours (ML-KNN) [7] and instance-based logistic regression (IBLR) [23] are two seminal instance-based multi-label algorithms. Binary relevance with support vector machines as a binary classifier (BR$\mathrm{SmO})$ is one of the best-performing algorithms $[2,35]$. For optimising the threshold for the other methods, we use OneThreshold, which optimises a single threshold on the selected evaluation metric [36]. For the extreme datasets, we compare the results of LCIF with the published results of FASTXML [11], a fast tree-based method for extreme multi-label classification.

For the state-of-the-art methods, we use the implementations available in the Mulan library [31]. We implemented LCIF in $\mathrm{C}++$ and made the source code publicly available ${ }^{1}$. We use 64 threads to compute similarities and predictions in parallel on a test server from 2013, which has two 8-core processors (Intel E5-2690) and 64 GB RAM. Remark that we cannot use Mulan (or Meka [32]) for extreme datasets since methods like BRSMO employ the binary relevance strategy for training $L$ binary classifiers, which is not feasible.

\footnotetext{
1 https://bitbucket.org/len_feremans/lcif
}

Evaluation Metrics Multi-label evaluation metrics can be organised in different ways. Example-based evaluation metrics are averaged over all instances. Label-based evaluation metrics look at the different ratios between true-positive, false-positive and false-negative predictions for each label. Label-based micro scores give each instance the same weight, while macro scores give each label the same weight, giving equal weight to frequent and infrequent labels. Within the Example-based category, we make the distinction between metrics based on the bipartition between relevant and non-relevant labels, metrics based on the ranking of confidence scores, and metrics based on the individual score for each label. We report the following example-based multi-label evaluation metrics:

\section{Definition 14 (Example-based Metrics)}

$$
\begin{gathered}
\text { Example-based Accuracy }=\frac{1}{N} \sum_{i=1}^{N} \frac{\left|\mathbf{y}_{\mathbf{i}} \cap \hat{\mathbf{y}}_{\mathbf{i}}\right|}{\left|\mathbf{y}_{\mathbf{i}} \cup \hat{\mathbf{y}}_{\mathbf{i}}\right|}, \\
\text { Hamming loss }=\frac{1}{N} \sum_{i=1}^{N} \frac{1}{L}\left|\mathbf{y}_{\mathbf{i}} \triangle \hat{\mathbf{y}}_{\mathbf{i}}\right|,
\end{gathered}
$$

where $N$ is the number of test instances, $\hat{\mathbf{y}}_{\mathbf{i}}$ is the predicted set of labels and $\mathbf{y}_{\mathbf{i}} \triangle \hat{\mathbf{y}}_{\mathbf{i}}$ is the symmetric difference (or XOR) of actual and predicted labels.

Definition 15 (Label-based Metrics) We define for each label $y_{k}$ the number of true positives, false negatives, false positives and corresponding metrics as

$$
\begin{aligned}
& t p=\sum_{i=1}^{N} \delta\left(y_{k} \in \mathbf{y}_{\mathbf{i}} \wedge y_{k} \in \hat{\mathbf{y}}_{\mathbf{i}}\right), \\
& f n=\sum_{i=1}^{N} \delta\left(y_{k} \in \mathbf{y}_{\mathbf{i}} \wedge y_{k} \notin \hat{\mathbf{y}}_{\mathbf{i}}\right), \\
& f p=\sum_{i=1}^{N} \delta\left(y_{k} \notin \mathbf{y}_{\mathbf{i}} \wedge y_{k} \in \hat{\mathbf{y}}_{\mathbf{i}}\right),
\end{aligned}
$$




$$
\begin{aligned}
\text { precision } & =\frac{t p}{t p+f p}, \quad \text { recall }=\frac{t p}{t p+f n}, \\
F 1 & =2 \cdot \frac{\text { precision } \cdot \text { recall }}{\text { precision }+ \text { recall }} .
\end{aligned}
$$

For label-based evaluation metrics, we report both micro and macro F1 metrics. Micro F1 is based on the previous definitions but based on totals of true positives, false negatives and false positives over all labels $L$. For macro F1, we first compute precision and recall for each label separately and then compute the average.

Definition 16 (Micro and Macro Precision) We define micro and macro precision (analogous for recall and F1) as:

$$
\begin{gathered}
\text { precision }_{\text {micro }}=\frac{\sum_{j=1}^{L} t p_{j}}{\sum_{j=1}^{L} t p_{j}+\sum_{j=1}^{L} f p_{j}}, \\
\text { precision }_{\text {macro }}=\frac{1}{L} \sum_{j=1}^{L} \frac{t p_{j}}{t p_{j}+f p_{j}} .
\end{gathered}
$$

For the extreme datasets, we omit hamming loss which was close to 0 given the extreme number of labels and is a less suitable metric in such settings [37]. Instead, we report precision@ $k$.

Definition 17 (Precision@k) We compute precision@k based on the $k$ predictions with the highest confidence score, defined as:

$$
\text { precision } @ k=\frac{1}{N \cdot k} \sum_{i=1}^{N} \sum_{y_{j} \in \mathbf{y}_{\mathbf{i}}} \delta\left(\operatorname{rank}\left(y_{j}, \hat{\mathbf{y}}_{\mathbf{i}}\right) \leq k\right),
$$

where $\operatorname{rank}\left(y_{j}, \hat{\mathbf{y}}_{\mathbf{i}}\right)$ returns the rank for label $y_{j}$ in the list of predictions sorted on descending confidence score.

Hyperparameter Tuning For each method, we have to optimise several hyperparameters. The parameter $k$ is often set to a fixed value in other research, or only iterated over a small set of possible values (e.g., 5, 10, 15). However, optimising $k$ can have a significant effect on reported evaluation metric values. Therefore, we vary $k$ for ML-KNN and IBLR between 1 and 59 in steps of 2 . For instance-based $\mathrm{kNN}$, we vary $k$ in steps of 50 , that is $k \in\{1,5,50,100, \ldots, 350\}$. We remark that the large values of $k$ are due to the similarity weighted scores and common within user-based collaborative filtering. We vary $\alpha$ en $\beta$ for the power transform of instanceand feature-based $\mathrm{kNN}$ in $\{0.5,1.0,1.5,2.0\}$. For LCIF, we vary $\lambda$ between 0.0 and 1.0 in steps of 0.1 . Remark that we re-use the neighbour (and similarity) matrix and only compute it once for the maximal value of $k$ speeding up grid search considerably. For optimising the single threshold $t$, we perform two passes: first, we vary $t$ between 0.0 and 1.0 in steps of 0.1 to obtain a temporary optimum $t_{\text {pass } 1}$, and then we take steps of 0.01 and vary between $t_{\text {pass } 1}-0.05$ and $t_{\text {pass } 1}+0.05$ to obtain the final value. For the state-of-the-art methods, this is implemented by OneThreshold in Mulan. We use the same procedure for LCIF but minimise the difference between predicted and actual label cardinality (see Section 3.4) instead of maximising a selected evaluation metric. Finally, for the extreme datasets, we employ feature selection and select the top $s$ features using entropy. We search for the optimal value of $s \in M \times\{0.01,0.1,0.25,0.5,0.75,0.99,1.0\}$. Note that we do not perform a full grid search, but instead first find the optimal value of $s$, assuming default parameters for other values (i.e., $k=100, \lambda=0.5, \alpha=\beta=1.0$ ). Next, we find the optimal value of $k$ assuming $\alpha=1.0$ and the value of $s$ previously found, then for $\alpha$ using the optimal $k$ and $s$, then for $\beta, \lambda$ and finally for $t$ using the previously computed parameters.

To have a stable estimate for hyperparameters selected using grid search, we perform 10-fold cross-validation for LCIF on the training set for the large datasets. After the 10-fold cross-validation search finishes, we select the average parameter combination that optimises the selected evaluation metric on the training data. For ML-KNN and IBLR, we report the optimal hyperparameters optimised directly on the test set, thereby making the Oracle assumption for performance reasons. For BR-Smo, we keep default parameters for the (linear) kernel function. For the extreme datasets, we skip 10fold cross-validation for performance reasons and instead perform grid search on a sample consisting of the first 10000 instances (1000 instances for Eurlex and Wiki10). We report results computed on the publicly available train-test splits.

\subsection{Classification Performance LCIF}

Here we discuss accuracy on different evaluation metrics for large and extreme datasets.

Large Datasets We compare our algorithms based on a variety of evaluation metrics for multi-label classification. This is common practice since different methods have different biases towards each metric [8]. Table 2 shows the results for each different evaluation metric on the large datasets for LCIF and the selected stateof-the-art multi-label classifiers. Results highlighted in bold perform best on the selected metric and dataset. Missing values for IBLR for the Delicious and $I M D B-F$ datasets are due to time-out on our test server. 
If we compare the ranking of all algorithms, we see that LCIF performs better than ML-KNN, IBLR and BR-SMO for both accuracy, micro F1 and macro F1. On hamming loss, BR-SMO performs best, but the difference with LCIF is small. We also see that instancebased kNN ranks second for both accuracy, micro F1 and macro F1 outperforming both ML-KNN and BRSMO. The feature-based kNN, by itself, does not perform great, but is comparable with ML-KNN, while requiring no $k$-nearest neighbour search at test time. Note that the current version of feature-based kNN is significantly better than the preliminary version described in [1]. Compared to the original version, we now compute the full similarity matrix and not only the top-k highest similarities and scale similarities using the parameter $\beta$.

Extreme Datasets For extreme multi-label classification (XML) we compare with the published results of FastXmL [11,33]. Table 3 shows the results on the extreme datasets. Results of micro or macro F1 are generally not available for other XML methods and are thus provided for information. LCIF performs better than FAstXmL on Eurlex, Wiki10 and WikiLSHTC,

Table 2 Comparing accuracy of LCIF with ML-KNN, IBLR and BR-SMO on the large datasets

\begin{tabular}{lcccccc} 
& $\begin{array}{r}\text { INS. } \\
\text { KNN }\end{array}$ & $\begin{array}{r}\text { FEAT. } \\
\text { KNN }\end{array}$ & LCIF & $\begin{array}{r}\text { ML- } \\
\text { KNN }\end{array}$ & IBLR & $\begin{array}{r}\text { BR- } \\
\text { SMO }\end{array}$ \\
\hline Accuracy $\uparrow$ & & & & & & \\
Medical & 0.563 & 0.601 & 0.636 & 0.421 & 0.442 & $\mathbf{0 . 6 9 9}$ \\
Corel5k & 0.163 & $\mathbf{0 . 1 7 4}$ & 0.170 & 0.147 & 0.105 & 0.098 \\
Bibtex & $\mathbf{0 . 3 4 7}$ & 0.218 & 0.341 & 0.208 & 0.174 & 0.321 \\
Delicious & $\mathbf{0 . 2 3 0}$ & 0.122 & $\mathbf{0 . 2 3 0}$ & 0.193 & $n / a$ & 0.130 \\
IMDB-F & $\mathbf{0 . 2 5 0}$ & 0.235 & $\mathbf{0 . 2 5 0}$ & 0.244 & $n / a$ & 0.005 \\
Avg. rank & 2.2 & 3.4 & $\mathbf{1 . 8}$ & 4.2 & 5.6 & 3.8 \\
\hline Micro F1 $\uparrow$ & & & & & & \\
Medical & 0.622 & 0.645 & 0.690 & 0.505 & 0.506 & $\mathbf{0 . 7 7 3}$ \\
Corel5k & 0.266 & 0.263 & $\mathbf{0 . 2 7 4}$ & 0.245 & 0.163 & 0.166 \\
Bibtex & 0.426 & 0.276 & $\mathbf{0 . 4 2 7}$ & 0.315 & 0.250 & 0.416 \\
Delicious & 0.368 & 0.214 & $\mathbf{0 . 3 6 9}$ & 0.322 & $n / a$ & 0.224 \\
IMDB-F & 0.341 & 0.337 & 0.346 & $\mathbf{0 . 3 5 8}$ & $n / a$ & 0.014 \\
Avg. rank & 2.6 & 4.0 & $\mathbf{1 . 4}$ & 3.6 & 5.8 & 3.6 \\
\hline Macro F1 $\uparrow$ & & & & & & \\
Medical & 0.339 & 0.447 & $\mathbf{0 . 4 9 2}$ & 0.245 & 0.247 & 0.457 \\
Corel5k & 0.315 & 0.308 & 0.315 & $\mathbf{0 . 3 2 6}$ & 0.161 & 0.317 \\
Bibtex & 0.321 & 0.129 & $\mathbf{0 . 3 2 8}$ & 0.170 & 0.147 & 0.315 \\
Delicious & 0.180 & 0.067 & $\mathbf{0 . 1 8 1}$ & 0.088 & $n / a$ & 0.098 \\
IMDB-F & 0.090 & 0.055 & $\mathbf{0 . 0 8 4}$ & 0.056 & $n / a$ & 0.011 \\
Avg. rank & 2.5 & 4.6 & $\mathbf{1 . 7}$ & 3.6 & 5.6 & 3.0 \\
\hline Hamming loss $\downarrow$ & & & & & \\
Medical & 0.025 & 0.026 & 0.021 & 0.024 & 0.029 & $\mathbf{0 . 0 1 2}$ \\
Corel5k & 0.014 & $\mathbf{0 . 0 1 0}$ & $\mathbf{0 . 0 1 0}$ & 0.022 & 0.027 & 0.012 \\
Bibtex & 0.017 & 0.017 & $\mathbf{0 . 0 1 4}$ & 0.020 & 0.021 & 0.016 \\
Delicious & 0.025 & 0.030 & 0.024 & 0.021 & $n / a$ & $\mathbf{0 . 0 1 8}$ \\
IMDB-F & 0.094 & 0.083 & 0.082 & 0.101 & $n / a$ & $\mathbf{0 . 0 7 2}$ \\
Avg. rank & 3.9 & 3.6 & 1.9 & 4.0 & 6.0 & $\mathbf{1 . 6}$ \\
\hline & & & & & & \\
\hline
\end{tabular}

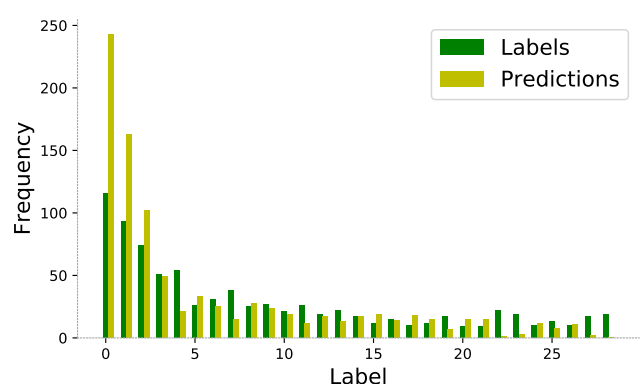

(a) Single threshold

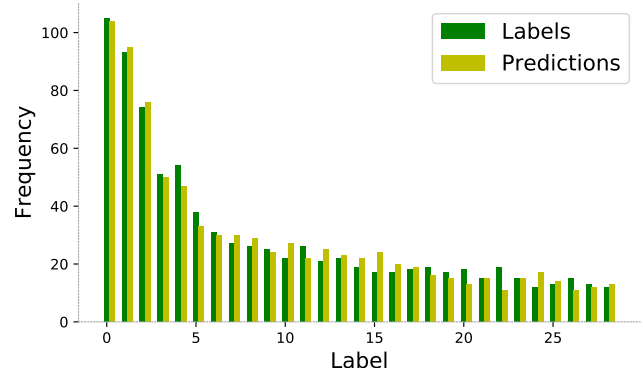

(b) Label-specific threshold

Fig. 1 Effect of thresholding on the distribution of actual versus predicted labels on the imbalanced dataset Corel5k

but not on AmazonCat. We remark that many other recent, rank-based optimised, XML methods exist [33]. However, in classification benchmarks, the best results are reported by ensemble methods that combine many models, possibly using different algorithms and feature representations. Comparing a single simple model with a complex ensemble-based method would not be informative. We conclude that our method produces excellent results on extreme datasets.

Imbalanced Datasets LCIF improves the accuracy on minority labels in imbalanced datasets. Firstly, we remark that feature-based cosine similarity is corrected for label imbalance since we normalise all label vectors to unit length during preprocessing. As such, weights for infrequent labels (and features) will be much higher. In Figure 1 we show the frequency of the top 30 most frequent labels (and predicted labels) on the Corel5k dataset where hyperparameters are optimised on the macro F1 metric during grid search. By using a single threshold, our method overestimates majority labels and underestimates minority labels. However, by using label-specific thresholds, we get a better match in the distribution of actual versus predicted labels. Here, we base the class-specific thresholds on the prior distribution of classes in the training dataset. We report an average gain of $1.1 \%$ on macro $\mathrm{F} 1$ on the large datasets. 
Table 3 Comparing accuracy of LCIF with FastXmL on the extreme datasets

\begin{tabular}{|c|c|c|c|c|}
\hline & $\begin{array}{l}\text { INs. } \\
\text { KNN } \\
\end{array}$ & $\begin{array}{r}\text { FEAT. } \\
\text { KNN } \\
\end{array}$ & LCIF & FASTXML \\
\hline \multicolumn{5}{|l|}{ Micro F1 $\uparrow$} \\
\hline Eurlex & 0.506 & 0.222 & 0.517 & $n / a$ \\
\hline Wiki10 & 0.359 & 0.274 & 0.358 & $n / a$ \\
\hline$R C V 1$ & 0.637 & 0.450 & 0.632 & $n / a$ \\
\hline AmazonCat & 0.611 & 0.418 & 0.625 & $n / a$ \\
\hline WikiLSHTC & 0.329 & 0.144 & 0.342 & $n / a$ \\
\hline \multicolumn{5}{|l|}{ Macro F1 $\uparrow$} \\
\hline Eurlex & 0.509 & 0.381 & 0.512 & $n / a$ \\
\hline Wiki10 & 0.324 & 0.261 & 0.324 & $n / a$ \\
\hline$R C V 1$ & 0.152 & 0.071 & 0.152 & $n / a$ \\
\hline AmazonCat & 0.463 & 0.285 & 0.463 & $n / a$ \\
\hline WikiLSHTC & 0.130 & 0.130 & 0.130 & $n / a$ \\
\hline \multicolumn{5}{|l|}{ Precision@1 个 } \\
\hline Eurlex & 0.763 & 0.407 & 0.776 & 0.713 \\
\hline Wiki10 & 0.832 & 0.722 & 0.832 & 0.830 \\
\hline$R C V 1$ & 0.830 & 0.762 & 0.840 & $n / a$ \\
\hline AmazonCat & 0.775 & 0.657 & 0.811 & 0.931 \\
\hline WikiLSHTC & 0.491 & 0.258 & 0.518 & 0.497 \\
\hline \multicolumn{5}{|l|}{ Precision@3 } \\
\hline Eurlex & 0.611 & 0.302 & 0.622 & 0.599 \\
\hline Wiki10 & 0.724 & 0.531 & 0.724 & 0.675 \\
\hline$R C V 1$ & 0.663 & 0.615 & 0.672 & $n / a$ \\
\hline AmazonCat & 0.665 & 0.589 & 0.698 & 0.782 \\
\hline WikiLSHTC & 0.317 & 0.178 & 0.336 & 0.331 \\
\hline \multicolumn{5}{|l|}{ Precision@5 个 } \\
\hline Eurlex & 0.504 & 0.244 & 0.514 & 0.504 \\
\hline Wiki10 & 0.637 & 0.469 & 0.637 & 0.578 \\
\hline$R C V 1$ & 0.480 & 0.449 & 0.487 & $n / a$ \\
\hline AmazonCat & 0.547 & 0.504 & 0.577 & 0.634 \\
\hline WikiLSHTC & 0.238 & 0.141 & 0.251 & 0.244 \\
\hline
\end{tabular}

\subsection{Runtime Performance InstanceKnnFast}

We now compare InSTANCEKNNFAST with the following state-of-the-art methods in top- $k$ query retrieval: TAAT without pruning, Fagin's Threshold Algorithm (FAGIN TA) and the in-memory variant of DAAT with Weak-AND pruning (M-WAND) [19,21].

Analysis Term-at-a-time First, we analyse the runtime behaviour of baseline $\mathrm{kNN}$ algorithm INSTANCEKnNSEARCH shown in Algorithm 2. This algorithm has three properties that make it efficient. We will use dataset characteristics from the extreme dataset WikiLSHTC for illustration (see Table 1). Firstly, we compute a sparse dot product between the query instance and each instance from the training dataset. In this dataset, there are $M \approx 1.6 \times 10^{6}$ features, however on average an instance has only 42 non-zero features, i.e., fcard is 42 . Clearly, computing a million of zero multiplications for the naive full dot product is wasteful. Using the TAAT traversal strategy, we only compute $x_{q, j} \cdot x_{i, j}$ terms that have a non-zero value for feature value $x_{i, j}$. Secondly, the inverted index causes a form of rudimen- tary pruning by only considering candidate instances $x_{i}$ having a non-zero feature in common with the query instance. We experimented on WikiLSHTC and computed the average number of candidates for 500 random test instances. We found that on average, only for $42 \%$ of instances the similarity is computed, thereby pruning about a million of instances from the training dataset (see Table 4). Thirdly, we also compute confidence scores in a sparse manner, thereby only computing non-zero terms for the confidence score for candidate labels, i.e., labels that occur for at least one neighbour. This is important since on average, each training instance has only 3 labels, i.e. lcard is 3.3 , while $L \approx 0.3 \times 10^{6}$.

Pruning Performance The runtime performance is dependent on pruning, i.e., the number of candidate instances for which we compute the cosine similarity. We compare the average number of candidate instances for each state-of-the-art method. We set $k$ to 100 and use the first 1000 test instances to compute this average. We remark that InSTANCEKNNFAST is identical to M-WAnd if $m=0$, and identical to InstanceKNN when $m$ is set high (such that $\mathcal{I}_{\text {daat }}=\emptyset$ ). For InSTANCEKNNFAST, we vary the hyperparameter $m \in$ $\{1,5,10,15,20,25,100\}$ (not $m=0$ ) and assume an Oracle that selects the best parameter.

The results are shown in Table 4 where we report both the absolute value and relative percentage of the average number of candidates. For the large datasets, all features are binary, however, after normalisation to unit length there is more variation in feature values, which is beneficial for pruning with our method. On the large datasets we see this effect, where InSTANCEKNNFAST evaluates fewer candidate instances compared to other techniques. For example, on the $I M D B-F$ dataset, we compute cosine similarity for $46 \%$ of the training instances for InstanceKnNSEARCH, 39\% for FAGIN TA, $28 \%$ for M-WAND and only $22 \%$ for INSTANCEKNNFAST. If we look at the extreme datasets, we find that M-WAND outperforms both FAGIN TA and INSTANCEKNNFAST on the majority of datasets. For small values of $m$, the number of instances in $I_{\text {taat }}$ is large, leading to a higher number of full evaluations. By considering only instances with a maximum feature value for low-density features, this could be resolved, but we will see in the next subsection, where we compare runtimes, that this is less important. Overall we conclude that INSTANCEKNNFAST for pruning is theoretically always better than or equal to M-WAND without partitioning and performs better than FAGIN TA and INSTANCEKNNSEARCH, under the assumptions of sparse datasets and high-dimensional queries. 
Table 4 Pruning of InstanceKnnFast and state-of-the-art top- $k$ query retrieval methods

\begin{tabular}{|c|c|c|c|c|c|c|}
\hline Dataset & & GIN TA & & I-WAND & INSTANCEKNNFAST & INSTANCEKNNSEARCH \\
\hline \multicolumn{7}{|c|}{ Avg number of candidate instances $\downarrow$} \\
\hline Medical & 197 & $(59 \%)$ & 214 & $(64 \%)$ & 214 (64\%) & $224(67 \%)$ \\
\hline Corel5k & 681 & $(14 \%)$ & 641 & $(14 \%)$ & $455(10 \%)$ & $719(16 \%)$ \\
\hline Bibtex & 4404 & $(90 \%)$ & 4553 & $(93 \%)$ & $4762(97 \%)$ & $4860(99 \%)$ \\
\hline Delicious & 3412 & $(26 \%)$ & 3120 & $(24 \%)$ & $3115(24 \%)$ & $4141 \quad(32 \%)$ \\
\hline$I M D B-F$ & 28389 & $(39 \%)$ & 20716 & $(28 \%)$ & $16170(22 \%)$ & $33509(46 \%)$ \\
\hline Eurlex & 14091 & $(91 \%)$ & 11785 & $(76 \%)$ & $12355(79 \%)$ & $15528(99 \%)$ \\
\hline Wiki10 & 13727 & $(97 \%)$ & 12592 & $(89 \%)$ & $14110(99 \%)$ & $14118(99 \%)$ \\
\hline$R C V 1$ & 305002 & $(48 \%)$ & 176038 & $(28 \%)$ & $196453(31 \%)$ & $569297 \quad(91 \%)$ \\
\hline AmazonCat & 195488 & $(11 \%)$ & 227326 & $(19 \%)$ & $290450(24 \%)$ & $741380(62 \%)$ \\
\hline WikiLSHTC & 462939 & $(26 \%)$ & 367645 & $(20 \%)$ & $594107 \quad(33 \%)$ & 899071 \\
\hline
\end{tabular}

Table 5 Runtime of InstanceKnnFast and state-of-the-art top-k query retrieval methods

\begin{tabular}{|c|c|c|c|c|c|c|c|}
\hline \multirow[t]{2}{*}{ Dataset } & \multirow{2}{*}{$\begin{array}{l}\text { M- } \\
\text { WAND }\end{array}$} & \multicolumn{5}{|c|}{ INSTANCEKNNFAST } & \multirow{2}{*}{$\begin{array}{r}\text { InSt. KNN } \\
\text { SEARCH }\end{array}$} \\
\hline & & $m=1$ & $m=20$ & $m=100$ & $m=500$ & $m=1000$ & \\
\hline \multicolumn{8}{|c|}{ Avg time $(\mathrm{ms})$ to retrieving top $\mathrm{kNN} \downarrow$} \\
\hline Eurlex & 17.4 & 12.8 & 1.4 & 1.3 & 1.4 & 1.4 & 1.5 \\
\hline Wiki10 & 62.4 & 1.8 & 1.6 & 1.5 & 1.6 & 1.7 & 1.7 \\
\hline$R C V 1$ & 103.7 & 97.4 & 43.8 & 32.6 & 32.6 & 33.9 & 37.5 \\
\hline AmazonCat & 215.4 & 174.1 & 45.1 & 37.7 & 38.7 & 45.1 & 45.2 \\
\hline WikiLSHTC & 171.1 & 94.3 & 35.7 & 27.7 & 27.9 & 27.6 & 28.6 \\
\hline
\end{tabular}

Runtime Performance We now compare our method with state-of-the-art-methods and report elapsed wall time. We do not report results for FAGIN TA since in our experiments we found that the random access cost and associated zero computations for computing full cosine similarity at each iteration caused much worse performance than the TAAT baseline without pruning. We report the number of milliseconds required for INSTANCEKnnSEARCH, M-WAnd and InstanceKnnFast with $m$ in $\{1,20,100,500,1000\}$. For each dataset, we take the first 1000 test instances and report the average time it takes to retrieve the exact set of 100 nearest neighbours using each algorithm. From the timings (averaged over 10 runs) we excluded time needed to load the data and create the inverted indexes since this took less than 1 minute on WikiLSHTC.

The results are shown in Table 5 . We omitted the results for the large datasets since the differences in milliseconds are too small. First, we remark that there is no clear one-to-one correspondence between pruning and runtime performance. Because of its simplicity and sparse optimisations, our current implementation of the INSTANCEKNNSEARCH method is far more efficient than M-WAND. M-WAND has overhead because of the computation and bookkeeping required for computing and comparing with the upper bound, such as the sort on document ID required to find the pivot. Note that both M-WAND and INSTANCEKNNFAST use the same code. We see that INSTANCEKNNFAST, when $m$ is set appropriately large, outperforms both state-of-the- art methods by a considerable margin on all extreme datasets. This is especially so for AmazonCat, where it is 6 times faster than M-WAND and 25\% faster than INSTANCEKNNSEARCH. We find that, on the one hand, when only a few instances in the inverted index match the current query, the overhead of computing and verifying the upper bound is probably not justified. On the other hand, when only low similarity instances remain, and there is a high likelihood for pruning, pruning becomes the faster alternative. We see this in AmazonCat, where for $m=100,98.6 \%$ of instances are indexed for TAAT traversal and $1.4 \%$ for DAAT traversal. However, during TAAT only $39 \%$ of instances are pruned (because of the inverted index), while during DAAT more than $99 \%$ of instances (in the remaining sample of about 16.000 instances) are pruned. We conclude that INSTANCEKNNFAST finds a natural balance between fast unpruned TAAT traversal and fast DAAT traversal with WAND pruning.

\subsection{Runtime Performance LCIF}

Large Datasets We now compare the total runtime required for both training and applying the model of LCIF and each of the state-of-the-art algorithms for large datasets. The results are shown in Table 6. For the large datasets, the difference in wall time is quite large as LCIF takes seconds or minutes where other methods take minutes or hours to complete. For Corel5k the relatively long runtime of 34.3 minutes for IBLR is due 
to learning the optimal weights using logistic regression for each label: training the optimal weights takes about 10 seconds per label but must be repeated for 374 labels. Due to this reason, IBLR was not able to complete on Delicious and $I M D B-F$. BR-Smo does finish for $D e-$ licious and $I M D B-F$ but runs for a full day when LCIF takes less than 1 minute. Table 6 also shows the runtime of instance- and feature-based kNN. The runtime of LCIF is approximately equal to the total runtime of the two components. We conclude that LCIF is orders of magnitude faster than ML-KNN, BR-SMO and IBLR.

Extreme Datasets In Table 7 we show the total time required by LCIF on the extreme datasets. We report subtotals for running instance-based $k$-nearest neighbours search, feature-based similarity matrix computation and feature-based predictions. For LCIF the total time is the sum of these steps. The remaining time needed for data loading, indexing, making instancebased predictions, combining predictions and computing and applying a single threshold is relatively small. Additionally, we report time to perform grid search for tuning hyperparameters. All experiments were run on a single test server with very moderate hardware specifications as described previously.

On WikiLSHTC, LCIF took less than 3 hours to finish on more than 500000 test instances, including grid search using a validation set of 10000 instances. Averaged over the number of test instances this means 22 ms per instance on average, of which the bulk is required for running the instance-based $k$-nearest neighbours search. Feature-based kNN predictions require less than 10 minutes in total and less than $1 \mathrm{~ms}$ per instance on average. In FASTXML the authors report wall times of 1.5 hours on WikiLSHTC for training only, depending on the hyperparameters [11]. However, some hyperparameters, such as the parameter that controls the number of iterations have a serious influence on both runtime and precision, and it is unclear how to optimise this and the 7 other hyperparameters efficiently. We conclude that our algorithm is very efficient and does hyperparameter tuning, training and predictions in a few hours on commodity hardware for extreme datasets, taking less than $22 \mathrm{~ms}$ per instance to predict labels.

\section{Related Work}

We have examined the most important related work in Section 1 and experimentally compared our method with existing state-of-the-art methods in Section 5. We now place our work into the wider context of multi-label
Table 6 Runtime results of LCIF and the state-of-the-art multi-label classifications methods on the large datasets

\begin{tabular}{lllllll} 
Dataset & $\begin{array}{c}\text { FEAT. } \\
\text { KNN }\end{array}$ & $\begin{array}{l}\text { INS. } \\
\text { KNN }\end{array}$ & LCIF & $\begin{array}{l}\text { ML- } \\
\text { KNN }\end{array}$ & IBLR & $\begin{array}{l}\text { BR- } \\
\text { SMO }\end{array}$ \\
\hline \multicolumn{7}{l}{ Total train } \\
and test time for classifier $\downarrow$ \\
\hline Medical & $0.1 \mathrm{~s}$ & $0.0 \mathrm{~s}$ & $0.1 \mathrm{~s}$ & $0.5 \mathrm{~s}$ & $1.5 \mathrm{~s}$ & $6.5 \mathrm{~s}$ \\
Corel5k & $0.0 \mathrm{~s}$ & $0.1 \mathrm{~s}$ & $0.1 \mathrm{~s}$ & $15.5 \mathrm{~s}$ & $34.3 \mathrm{~m}$ & $6.5 \mathrm{~m}$ \\
Bibtex & $1.9 \mathrm{~s}$ & $0.3 \mathrm{~s}$ & $2.6 \mathrm{~s}$ & $1.7 \mathrm{~s}$ & $8.2 \mathrm{~m}$ & $10.1 \mathrm{~m}$ \\
Delicious & $0.6 \mathrm{~s}$ & $0.2 \mathrm{~s}$ & $1.3 \mathrm{~s}$ & $3.3 \mathrm{~m}$ & $n / a$ & $14.0 \mathrm{~h}$ \\
$I M D B-F$ & $13.5 \mathrm{~s}$ & $33.8 \mathrm{~s}$ & $49.2 \mathrm{~s}$ & $2.1 \mathrm{~h}$ & $n / a$ & $29.4 \mathrm{~h}$ \\
\hline
\end{tabular}

Table 7 Runtime results of LCIF on the extreme datasets

\begin{tabular}{llllll} 
Dataset & $\begin{array}{l}\text { Grid } \\
\text { search }\end{array}$ & INS. KNN & \multicolumn{2}{l}{ FEAT. KNN } & LCIF \\
search & simil & predict & total \\
\hline \multicolumn{5}{l}{ Total time for classifier $\downarrow$} \\
\hline Eurlex & $51.4 \mathrm{~s}$ & $4.6 \mathrm{~s}$ & $0.8 \mathrm{~s}$ & $12.7 \mathrm{~s}$ & $24.4 \mathrm{~s}$ \\
Wiki10 & $9.9 \mathrm{~m}$ & $9.8 \mathrm{~s}$ & $26.4 \mathrm{~s}$ & $3.6 \mathrm{~m}$ & $5.1 \mathrm{~m}$ \\
RCV1 & $8.2 \mathrm{~m}$ & $58.9 \mathrm{~m}$ & $6.0 \mathrm{~s}$ & $2.9 \mathrm{~m}$ & $63.0 \mathrm{~m}$ \\
AmazonCat & $11.6 \mathrm{~m}$ & $136.3 \mathrm{~m}$ & $28.8 \mathrm{~s}$ & $5.7 \mathrm{~m}$ & $145.2 \mathrm{~m}$ \\
WikiLSHTC & $11.8 \mathrm{~m}$ & $154.8 \mathrm{~m}$ & $31.1 \mathrm{~s}$ & $8.3 \mathrm{~m}$ & $167.9 \mathrm{~m}$ \\
\hline
\end{tabular}

classification.

Instance-based Learning Several instance-based learning methods for multi-label classification have been developed. ML-KNN was one of the first methods [7]. In ML-KNN the authors first apply traditional kNN, using Euclidean distance. Next, they count the number of times each label occurs in the neighbourhood. Then they apply the maximum a posteriori principle for each label independently to determine if a label is relevant or not. They estimate prior probabilities by computing $\mathrm{kNN}$ for each training instance and then compute these probabilities for each label. In theory, ML-KNN could also adopt an inverted index and sparse computation of similarities, probabilities and predictions. However, the complexity is worse than that of LCIF, which is $\mathcal{O}(N \times M)$ for the $\mathrm{kNN}$ search and $\mathcal{O}(N \times N \times M)$ steps for computing probabilities. The authors experimentally show that ML-KNN outperformed other techniques, such as RANK-SvM on different example-based evaluation metrics. A possible disadvantage of ML-KNN is that it does not take label dependencies into account, which was addressed by subsequent research into dependent multi-label $k$-nearest neighbours [38].

In combining instance-based learning and logistic regression for multi-label classification (IBLR) the $k$ nearest neighbours are computed using Euclidean distance [23]. Then the authors use label counts from the nearest neighbours as a feature vector and apply logistic regression to learn the optimal hyper-plane for each label. This approach comes down to stacking and does account with dependencies between labels since all label counts are used as input for the logistic re- 
gression. However, applying logistic regression for each label independently does take considerable resources, as shown in our experiments, where the implementation from Mulan was unable to finish on some large datasets. Both ML-KNN and IBLR are considered stateof-the-art methods [8].

Spyromitros et al. propose BRKNN, where $\mathrm{kNN}$ is combined with the binary relevance method [26]. Like BRKNN, we compute the $k$-nearest neighbours once, independently of the number of labels. The authors also implement two extensions: BRKNN-a and BRKNNb. The BRKNN-b variant minimises the label cardinality between predicted and actual label sets, while BRKNNa returns the highest-scoring label as relevant, even if this label is below the threshold since for most benchmark multi-label datasets an empty set of labels is rare. Both ideas are implemented by our method. Compared to instance-based $\mathrm{kNN}$, BRKNN uses a different scoring function, which is the fraction of labels found in the $k$-nearest neighbours. Also, BRKNN does not make use of an inverted index. In future work, it could be interesting to experimentally validate different variations of instance-based prediction functions.

Wang et al. propose the Enhanced $\mathrm{kNN}$ algorithm (EKNN), that uses a weighted prediction function similar to instance-based kNN, but based on BM25 similarity and a more elaborate thresholding scheme. EKNN scored first in the challenge on large scale hierarchical text classification on example-based accuracy and F1 $[39,40]$. However, EkNN has a larger range of hyperparameters (both for BM25 and thresholding) to tune and is only applicable for text categorisation. The implementation of EKNN is based on an inverted index, similar to INSTANCEKNNSEARCH.

Imbalanced Datasets Different authors have studied how to improve the accuracy of imbalanced datasets. Sмоте is an algorithm for synthetic oversampling of multi-class instances with minority labels [41]. In preliminary experiments, we tried to adopt SMOTE for oversampling instances with minority labels together with downsampling of majority labels to generate balanced datasets. However, this did not significantly improve results on macro F1. Moreover, SMOTE has additional parameters for each minority label, making adoption challenging.

Tan proposes NWKNN, a neighbour-weighted kNN algorithm that achieves a significant performance improvement for text categorisation on imbalanced datasets [42]. Like NwKNN, our instance-based kNN method performs distance weighting and a power transform. Unlike NWKNN, we do not take the size of the member- ship of labels into account in the instance-based confidence score.

Liu et al. present a hybrid coupled $k$-nearest neighbour classification algorithm ( $\mathrm{HC}-\mathrm{KNN}$ ) for mixed-type data [43]. They employ feature weighting proportional to the number of feature-label co-occurrences and inversely proportional to the global label frequency. This is related to feature-based cosine similarity since we measure the feature-label co-occurrences by computing the cosine similarity between each feature and label column-wise and by normalising label vectors to unit length, we are dividing by the global label frequency (assuming binary positive data). The instance-based cosine similarity is related to the inter-coupled similarity measure if we employ one-hot-encoding of categorical features during preprocessing.

There is no related work on instance-based multilabel classification optimised for imbalanced extreme datasets. For example, Liu et al. propose an optimisation procedure to learn the correspondence between each feature value and label, but this has a complexity of $O\left(M^{3} \cdot L\right)$ [43]. Therefore, we propose to adopt a label-specific threshold to improve results on macro F1 [30]. However, we acknowledge that given a long tail of minority labels (e.g. occurring less than 5 times), high precision and recall remain challenging.

Fast Nearest Neighbours We did not consider combining TAAT traversal with pruning [21]. For example, we could prune entire dimensions using max_score pruning. While this technique is useful for pruning more instances, it remains uncertain if this would decrease the overall wall time for high-dimensional sparse datasets as is the case with INSTANCEKNNFAST. We also did not consider approximate kNN strategies used by other authors in extreme multi-label classification [10,44]. Since our algorithm can compute the exact set of $k$-nearest neighbours efficiently on extreme datasets, approximations are of less interest.

\section{Conclusion}

Inspired by recent work in recommender systems research, i.e., user-based and item-based collaborative filtering, we propose LCIF, a new algorithm for multi-label classification. Our predictions are based on the labels of the nearest neighbours in the training dataset. The instance-based method finds the top $k$ instances that are most similar using the features of the current test instance. The feature-based method gives higher weight to labels that are the most similar to each feature, where similarity is defined column-wise over all instances. A linear combination of the similarity weighted instance- 
and feature-based neighbourhood is computed to make the final prediction.

We created an efficient algorithm for finding the $k$ nearest neighbours using an inverted index and efficient sparse computation of cosine similarities and predictions. We extend this algorithm and create an even faster $k$-nearest neighbours search algorithm, by partitioning instances and combining term-at-a-time and document-at-a-time traversal with a tighter upper bound for Weak-AND pruning. We validated that this method can be $25 \%$ faster than the baseline method, and up to 6 times faster than existing top- $k$ query retrieval algorithms, assuming high-dimensional sparse datasets. LCIF requires only seconds to complete on large datasets, where classic methods take minutes or hours. For extreme datasets, we require less than 20 milliseconds per instance to predict labels on commodity hardware.

Experiments on ten real-world multi-label datasets from different domains, i.e., text categorisation, scene classification and social tagging domain, show that LCIF outperforms state-of-the-art algorithms such as multilabel $\mathrm{kNN}$, instance-based logistic regression and binary relevance with support vector machines on accuracy, micro F1 and macro F1. LCIF also produces excellent results on extreme datasets compared to FAstXML. Because of its efficiency at both train and test time, the possibility to generate explainable results, and excellent evaluation accuracy, LCIF is interesting for any extreme multi-label application, especially when making a trade-off between model/computational complexity and performance improvement. The source code of LCIF is publicly available and enables end-users to perform accurate extreme multi-label classification without the need for expensive clusters.

In future work, we see potential to improve further extreme multi-label learning algorithms inspired by advances in the related field of collaborative filtering. We also see potential to boost the prediction accuracy of LCIF, for example, by creating ensembles using boosting or stacking and adopting embedding algorithms, such as word or sentence embeddings learned using neural networks $[45,46]$.

\section{References}

1. L. Feremans, B. Cule, C. Vens, and B. Goethals, "Combining instance and feature neighbors for efficient multilabel classification," in 2017 IEEE International Conference on Data Science and Advanced Analytics (DSAA). IEEE, 2017, pp. 109-118.

2. G. Tsoumakas and I. Katakis, "Multi-label classification: An overview," International Journal of Data Warehousing and Mining, vol. 3, no. 3, 2006.
3. J. Read, B. Pfahringer, G. Holmes, and E. Frank, "Classifier chains for multi-label classification," Machine learning, vol. 85, no. 3, pp. 333-359, 2011.

4. R. E. Schapire and Y. Singer, "Improved boosting algorithms using confidence-rated predictions," Machine learning, vol. 37, no. 3, pp. 297-336, 1999.

5. C. Vens, J. Struyf, L. Schietgat, S. Džeroski, and H. Blockeel, "Decision trees for hierarchical multi-label classification," Machine Learning, vol. 73, no. 2, pp. 185-214, 2008.

6. A. Elisseeff, J. Weston et al., "A kernel method for multilabelled classification," in NIPS, vol. 14, 2001, pp. 681687.

7. M.-L. Zhang and Z.-H. Zhou, "Ml-knn: A lazy learning approach to multi-label learning," Pattern recognition, vol. 40, no. 7, pp. 2038-2048, 2007.

8. E. Gibaja and S. Ventura, "Multi-label learning: a review of the state of the art and ongoing research," Wiley Interdisciplinary Reviews: Data Mining and Knowledge Discovery, vol. 4, no. 6, pp. 411-444, 2014.

9. K. Bhatia, H. Jain, P. Kar, M. Varma, and P. Jain, "Sparse local embeddings for extreme multi-label classification," in Advances in Neural Information Processing Systems, 2015, pp. 730-738.

10. Y. Tagami, "Annexml: Approximate nearest neighbor search for extreme multi-label classification," in Proceedings of the 23rd ACM SIGKDD international conference on knowledge discovery and data mining. ACM, 2017, pp. 455-464.

11. Y. Prabhu and M. Varma, "Fastxml: A fast, accurate and stable tree-classifier for extreme multi-label learning," in Proceedings of the 20th ACM SIGKDD international conference on Knowledge discovery and data mining. ACM, 2014, pp. 263-272.

12. P. Resnick, N. Iacovou, M. Suchak, P. Bergstrom, and J. Riedl, "Grouplens: an open architecture for collaborative filtering of netnews," in Proceedings of the 1994 ACM conference on Computer supported cooperative work. ACM, 1994, pp. $175-186$.

13. J. S. Breese, D. Heckerman, and C. Kadie, "Empirical analysis of predictive algorithms for collaborative filtering," in Proceedings of the Fourteenth conference on Uncertainty in artificial intelligence. Morgan Kaufmann Publishers Inc., 1998, pp. 43-52.

14. B. Sarwar, G. Karypis, J. Konstan, and J. Riedl, "Item-based collaborative filtering recommendation algorithms," in Proceedings of the 10th international conference on World Wide Web. ACM, 2001, pp. 285-295.

15. R. J. Bayardo, Y. Ma, and R. Srikant, "Scaling up all pairs similarity search," in Proceedings of the 16th international conference on World Wide Web. ACM, 2007, pp. $131-140$.

16. A. Awekar and N. F. Samatova, "Fast matching for all pairs similarity search," in Web Intelligence and Intelligent Agent Technologies, 2009. WI-IAT'09. IEEE/WIC/ACM International Joint Conferences on, vol. 1. IEEE, 2009, pp. 295-300.

17. C. Xiao, W. Wang, X. Lin, and H. Shang, "Top-k set similarity joins," in Data Engineering, 2009. ICDE'09. IEEE 25th International Conference on. IEEE, 2009, pp. 916927.

18. D. C. Anastasiu and G. Karypis, "Fast parallel cosine k-nearest neighbor graph construction," in Proceedings of the Sixth Workshop on Irregular Applications: Architectures and Algorithms. IEEE Press, 2016, pp. 50-53.

19. R. Fagin, A. Lotem, and M. Naor, "Optimal aggregation algorithms for middleware," Journal of computer and system sciences, vol. 66, no. 4, pp. 614-656, 2003. 
20. S. Ding and T. Suel, "Faster top-k document retrieval using block-max indexes," in Proceedings of the 34th international ACM SIGIR conference on Research and development in Information Retrieval. ACM, 2011, pp. 993-1002.

21. M. Fontoura, V. Josifovski, J. Liu, S. Venkatesan, X. Zhu, and J. Zien, "Evaluation strategies for top-k queries over memory-resident inverted indexes," Proceedings of the VLDB Endowment, vol. 4, no. 12, pp. 1213-1224, 2011.

22. A. Z. Broder, D. Carmel, M. Herscovici, A. Soffer, and J. Zien, "Efficient query evaluation using a two-level retrieval process," in Proceedings of the twelfth international conference on Information and knowledge management. ACM, 2003, pp. 426-434.

23. W. Cheng and E. Hüllermeier, "Combining instancebased learning and logistic regression for multilabel classification," Machine Learning, vol. 76, no. 2-3, pp. 211225, 2009.

24. J. Wang, A. P. De Vries, and M. J. Reinders, "Unifying user-based and item-based collaborative filtering approaches by similarity fusion," in Proceedings of the 29th annual international ACM SIGIR conference on Research and development in information retrieval. ACM, 2006, pp. 501-508.

25. Y. Liu, "Crafting concurrent data structures," $\mathrm{PhD}$ dissertation, Lehigh University, 2015.

26. E. Spyromitros, G. Tsoumakas, and I. Vlahavas, "An empirical study of lazy multilabel classification algorithms," in Hellenic conference on Artificial Intelligence. Springer, 2008, pp. 401-406.

27. K. Verstrepen and B. Goethals, "Unifying nearest neighbors collaborative filtering," in Proceedings of the 8th ACM Conference on Recommender systems. ACM, 2014, pp. 177-184.

28. I. Triguero and C. Vens, "Labelling strategies for hierarchical multi-label classification techniques," Pattern Recognition, vol. 56, pp. 170-183, 2016.

29. Y. Yang, "A study of thresholding strategies for text categorization," in Proceedings of the 24 th annual international ACM SIGIR conference on Research and development in information retrieval. ACM, 2001, pp. 137-145.

30. K. Draszawka and J. Szymański, "Thresholding strategies for large scale multi-label text classifier," in 2013 6th International Conference on Human System Interactions (HSI). IEEE, 2013, pp. 350-355.

31. G. Tsoumakas, E. Spyromitros-Xioufis, J. Vilcek, and I. Vlahavas, "Mulan: A java library for multi-label learning," Journal of Machine Learning Research, vol. 12, no. Jul, pp. 2411-2414, 2011.

32. J. Read, P. Reutemann, B. Pfahringer, and G. Holmes, "Meka: a multi-label/multi-target extension to weka," The Journal of Machine Learning Research, vol. 17, no. 1, pp. 667-671, 2016.

33. K. Bhatia, K. Dahiya, H. Jain, Y. Prabhu, and M. Varma, "The extreme classification repository: multi-label datasets \& code," URL http://manikvarma. org/downloads/XC/XMLRepository. html, 2016.

34. I. Partalas, A. Kosmopoulos, N. Baskiotis, T. Artieres, G. Paliouras, E. Gaussier, I. Androutsopoulos, M.-R. Amini, and P. Galinari, "Lshtc: A benchmark for largescale text classification," arXiv preprint arXiv:1503.08581, 2015.

35. J. C. Platt, "12 fast training of support vector machines using sequential minimal optimization," Advances in kernel methods, pp. 185-208, 1999.

36. J. Read, B. Pfahringer, and G. Holmes, "Multi-label classification using ensembles of pruned sets," in Data Mining, 2008. ICDM'08. Eighth IEEE International Conference on. IEEE, 2008, pp. 995-1000.

37. H. Jain, Y. Prabhu, and M. Varma, "Extreme multi-label loss functions for recommendation, tagging, ranking \& other missing label applications," in Proceedings of the 22nd ACM SIGKDD International Conference on Knowledge Discovery and Data Mining. ACM, 2016, pp. 935944.

38. Z. Younes, F. Abdallah, and T. Denœux, "Multi-label classification algorithm derived from k-nearest neighbor rule with label dependencies," in Signal Processing Conference, 2008 16th European. IEEE, 2008, pp. 1-5.

39. X.-l. Wang, H. Zhao, and B. Lu, "Enhanced knearest neighbour algorithm for largescale hierarchical multi-label classification," in Proceedings of the Joint ECML/PKDD PASCAL Workshop on Large-Scale Hierarchical Classification, Athens, Greece, vol. 5, 2011.

40. I. Partalas, A. Kosmopoulos, N. Baskiotis, T. Artières, G. Paliouras, É. Gaussier, I. Androutsopoulos, M. Amini, and P. Gallinari, "LSHTC: A benchmark for large-scale text classification," CoRR, vol. abs/1503.08581, 2015.

41. N. V. Chawla, K. W. Bowyer, L. O. Hall, and W. P. Kegelmeyer, "Smote: synthetic minority oversampling technique," Journal of artificial intelligence research, vol. 16, pp. 321-357, 2002.

42. S. Tan, "Neighbor-weighted k-nearest neighbor for unbalanced text corpus," Expert Systems with Applications, vol. 28, no. 4, pp. 667-671, 2005.

43. C. Liu, L. Cao, and S. Y. Philip, "A hybrid coupled k-nearest neighbor algorithm on imbalance data," in 2014 International Joint Conference on Neural Networks (IJCNN). IEEE, 2014, pp. 2011-2018.

44. R. B. Zadeh and A. Goel, "Dimension independent similarity computation." Journal of Machine Learning Research, vol. 14, no. 1, pp. 1605-1626, 2013.

45. T. Mikolov, K. Chen, G. Corrado, and J. Dean, "Efficient estimation of word representations in vector space," arXiv preprint arXiv:1301.3781, 2013.

46. J. Devlin, M.-W. Chang, K. Lee, and K. Toutanova, "Bert: Pre-training of deep bidirectional transformers for language understanding," arXiv preprint arXiv:1810.04805, 2018. 